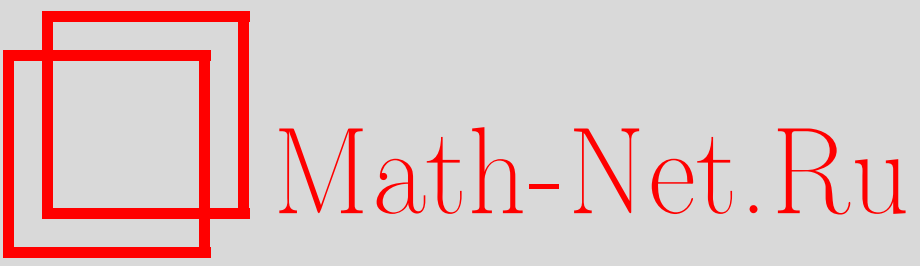

И. Ю. Осмоловский, Об оценке точности аппроксимации для асимптотических разложений в многомерном случае, Теория вероятн. и ее примен., 2009, том 54, выпуск 1, 154-160

DOI: https://doi.org/10.4213/tvp2551

Использование Общероссийского математического портала Math-Net.Ru подразумевает, что вы прочитали и согласны с пользовательским соглашением

http: //www . mathnet.ru/rus/agreement

Параметры загрузки:

IP: 3.85 .7 .115

26 апреля 2023 г., 11:33:57

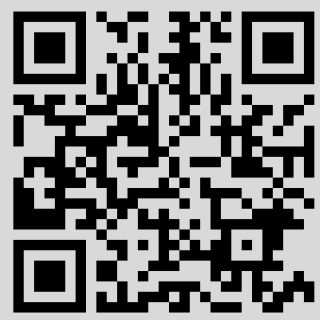




\title{
ОБ ОЦЕНКЕ ТОЧНОСТИ АППРОКСИМАЦИИ ДЛЯ АСИМПТОТИЧЕСКИХ РАЗЛОЖЕНИЙ В МНОГОМЕРНОМ СЛУЧАЕ
}

\begin{abstract}
Получена явная оценка остаточной части для одного асимптотического разложения в центральной предельной теореме для случайных величин, принимающих значения в многомерных пространствах.
\end{abstract}

Ключевье слова и фразы: центральная предельная теорема, асимптотические разложения, точность аппроксимации.

В [2] было получено асимптотическое разложение для плотностей распределений нормированных сумм независимых одинаково распределенных случайных величин, принимающих значения в многомерном евклидовом пространстве, при условии, что момент нормы порядка $m+2$, где $m \geqslant 2$ - натуральные, конечен. Оценка остаточной части этого разложения содержит слагаемые $O\left(n^{-(m+1) / 2}\right), n \rightarrow \infty$. Цель настоящей работы - получить явную оценку остаточной части разложения.

Пусть $X_{1}, X_{2}, \ldots$ - независимые одинаково распределенные величины с нулевым средним и единичным ковариационным оператором, принимающие значения в $d$-мерном евклидовом пространстве $E^{d}$, и $P-$ распределение $X_{1}$. Пусть для характеристической функции $f(t)$ распределения $P$ выполнено условие

$$
\int_{E^{d}}|f(t)|^{\nu} d t<\infty
$$

при некотором $\nu>0$, и пусть для некоторой пары $(\mu, T)$, где $e^{-|t|^{2} / 2} \leqslant \mu(t) \leqslant 1$ и $T>0$, при всех $|t| \leqslant T$ выполняется неравенство $|f(t)| \leqslant \mu(t)$.

Нам понадобятся величины

$$
B_{k}=\frac{1}{(2 \pi)^{d}} \int_{E^{d}}|t|^{k} e^{-|t|^{2} / 2} d t, \quad B_{k, n}=\frac{1}{(2 \pi)^{d}} \int_{|t| \leqslant T \sqrt{n}}|t|^{k} \mu^{n}\left(\frac{t}{\sqrt{n}}\right) d t .
$$

Величина $B_{k}$ асимптотически (при $d \rightarrow \infty$ ) равна $\frac{1}{2} \pi^{-d / 2} d^{k / 2}$. Для распределения с конечным четвертым моментом пару $(\mu, T)$ можно подобрать так, чтобы $B_{k, n} \rightarrow B_{k}$ при $n \rightarrow \infty$ для любого фиксированного $k>0$.

Обозначим $P_{n}$ распределение нормированной суммы $\left(X_{1}+\cdots+X_{n}\right) / \sqrt{n}$. При выполнении условия (1) для всех $n \geqslant \nu$ существует плотность $p_{n}(x), x \in E^{d}$, распределения $P_{n}$.

Пусть $\Phi(x)$ - нормальный закон с нулевым средним и единичным ковариационным оператором в $E^{d}$, а $\varphi(x)-$ его плотность. Для $e \in E^{d},|e|=1$, и натуральных $k$ обозначим

$$
\beta_{k}(e)=\beta_{k}(e, P):=\int_{E^{d}}|(e, u)|^{k} P(d u), \quad \beta_{k}=\sup _{|e|=1} \beta_{k}(e) .
$$

Здесь $(\cdot, \cdot)$ означает скалярное произведение, а $|\cdot|-$ норму вектора в $E^{d}$. Введем также обозначение

$$
\bar{\beta}_{k}=\int_{E^{d}}|(e, u)|^{k} \Phi(d u)
$$

* Московский государственный университет им. М.В. Ломоносова, механикоматематический факультет, кафедра теории вероятностей, Ленинские горы 1, 119992 Москва, Россия; e-mail: igosm@mail.ru 
Последний интеграл не зависит от направления вектора $e \in E^{d},|e|=1$, величина $\bar{\beta}_{k}$ совпадает с $k$-м моментом одномерного стандартного нормального закона. Положим

$$
\begin{gathered}
\Delta_{k}(e)=\int_{E^{d}}(e, u)^{k}(P-\Phi)(d u), \quad \delta_{k}=\sup _{|e|=1}\left|\Delta_{k}(e)\right|, \\
D_{3}=\sum_{s=3}^{m+1} \frac{\delta_{s}}{s !}, \quad D_{3, T}=\sum_{s=3}^{m+1} \frac{\delta_{s}}{s !} T^{s-3} .
\end{gathered}
$$

Лемма. В указанных выше обозначениях и предположениях, если величина $\beta_{m+2}$ конечна, то при $n \geqslant \max (\nu, 2 m)$ существует плотность $p_{n}(x)$ и для любого $x \in E^{d}$ справедливо равенство

$$
\begin{aligned}
& p_{n}(x)-\varphi(x)=\frac{1}{(2 \pi)^{d}} \\
& \quad \times \int_{|t| \leqslant T \sqrt{n}} e^{-i(t, x)} e^{-|t|^{2} / 2} \sum_{k=1}^{m} C_{n}^{k} e^{k|t|^{2} /(2 n)}\left(\sum_{s=3}^{m+1} \frac{i^{s} \Delta_{s}\left(e_{t}\right)}{s !}\left|\frac{t}{\sqrt{n}}\right|^{s}\right)^{k} d t+R,
\end{aligned}
$$

где $e_{t}=t /|t| u$

$$
\begin{gathered}
\| R \mid \leqslant \frac{\beta_{m+2}+\bar{\beta}_{m+2}}{(m+2) !} \sum_{k=1}^{m+1} \frac{D_{3, T}^{k-1}}{n^{(m+k-1) / 2}} \frac{B_{3(k-1)+m+2, n-k}}{k !}+\frac{D_{3, T}^{m+1}}{n^{(m+1) / 2}} \frac{B_{3(m+1), n-(m+1)}}{(m+1) !} \\
+\frac{n^{d / 2}}{(2 \pi)^{d}} \alpha^{n-\nu}(T) \int_{|t| \geqslant T}|f(t)|^{\nu} d t+\frac{1}{(2 \pi)^{d}} \int_{|t| \geqslant T \sqrt{n}} e^{-|t|^{2} / 2} d t, \\
\alpha(T)=\max \{f(t):|t| \geqslant T\}<1 .
\end{gathered}
$$

Доказательство этой леммы фактически повторяет доказательство соответствующей леммы из [2] и доказательство теорем из [3, §14], поэтому мы его опускаем.

Из утверждения леммы следует, что с точностью до величины $R$, указанной в ее формулировке, разность $p_{n}(x)-\varphi(x)$ есть

$$
\begin{aligned}
& \sum_{k=1}^{m} \frac{1}{(2 \pi)^{d}} \int_{|t| \leqslant T \sqrt{n}} e^{-i(t, x)} e^{-|t|^{2} / 2} C_{n}^{k} e^{k|t|^{2} /(2 n)}\left(\sum_{s=3}^{m+1} \frac{i^{s} \Delta_{s}\left(e_{t}\right)}{s !}\left|\frac{t}{\sqrt{n}}\right|^{s}\right)^{k} d t \\
& =\sum_{k=1}^{m} \sum_{k_{3}, \ldots, k_{m+1}} \frac{1}{(2 \pi)^{d}} \int_{|t| \leqslant T \sqrt{n}} e^{-i(t, x)} e^{-|t|^{2} / 2} C_{n}^{k} e^{k|t|^{2} /(2 n)} \frac{k !}{k_{3} ! \cdots k_{m+1} !} \\
& \quad \times\left(\frac{i^{3} \Delta_{3}\left(e_{t}\right)}{3 !}\left|\frac{t}{\sqrt{n}}\right|^{3}\right)^{k_{3}} \cdots\left(\frac{i^{m+1} \Delta_{m+1}\left(e_{t}\right)}{(m+1) !}\left|\frac{t}{\sqrt{n}}\right|^{m+1}\right)^{k_{m+1}} d t
\end{aligned}
$$

где внутреннее суммирование ведется по всем наборам неотрицательных целых чисел $k_{3}, \ldots, k_{m+1}$ таким, что $k_{3}+\cdots+k_{m+1}=k$. Зависимость наборов $k_{3}, \ldots, k_{m+1}$ от $k$ мы не указываем, это не должно вызывать недоразумений. Для данного $k$ число этих наборов $k_{3}, \ldots, k_{m+1}$ равно $C_{k+m-2}^{m-2}$.

Правую часть (3) можно переписать в виде

$$
\begin{aligned}
& \sum_{k=1}^{m} \sum_{k_{3}, \ldots, k_{m+1}} \frac{i^{l}}{(2 \pi)^{d}} \int_{|t| \leqslant T \sqrt{n}} e^{-i(t, x)} e^{-|t|^{2} / 2} e^{k|t|^{2} /(2 n)} \frac{n !}{(n-k) ! k_{3} ! \cdots k_{m+1} !} \\
& \quad \times\left(\frac{\Delta_{3}\left(e_{t}\right)}{3 !}\left|\frac{t}{\sqrt{n}}\right|^{3}\right)^{k_{3}} \cdots\left(\frac{\Delta_{m+1}\left(e_{t}\right)}{(m+1) !}\left|\frac{t}{\sqrt{n}}\right|^{m+1}\right)^{k_{m+1}} d t,
\end{aligned}
$$

где $l=3 k_{3}+\cdots+(m+1) k_{m+1}$. $n \rightarrow \infty$

Слагаемые в (4), которые соответствуют наборам $k_{3}, \ldots, k_{m+1}$ таким, что при

$$
\frac{n !}{(n-k) !} \frac{1}{n^{l / 2}}=O\left(\frac{1}{n^{(m+1) / 2}}\right)
$$


мы включим в остаточную часть разложения $p_{n}$. Эти слагаемые при росте $n$ убывают как $1 / n^{(m+1) / 2}$ или быстрее.

Таким образом, главная часть разложения $p_{n}$ будет формироваться только суммой (назовем ее основной) по $k=1, \ldots, m$ и по таким наборам $k_{3}, \ldots, k_{m+1}$, для которых $k_{3}+\cdots+k_{m+1}=k$ и $l / 2-k \leqslant m / 2$, последнее условие равносильно неравенству

$$
3 k_{3}+\cdots+(m+1) k_{m+1} \leqslant m+2 k .
$$

Отметим, что в основную сумму войдут все слагаемые из (4), связанные с $k=1$, и войдет лишь одно слагаемое, связанное с $k=m$ (оно соответствует набору $k_{3}, \ldots, k_{m+1}$, для которого $\left.k_{3}=m, k_{4}=\cdots=k_{m+1}=0, m \geqslant 3\right)$.

Обозначим через $R^{(m / 2)}$ сумму слагаемых из (4) по наборам $k_{3}, \ldots, k_{m+1}$, для которых не выполнено условие (5); для таких наборов

$$
k_{3}+\cdots+k_{m+1}=k, \quad 3 k_{3}+\cdots+(m+1) k_{m+1} \geqslant m+2 k+1,
$$

$$
\begin{aligned}
R^{(m / 2)}= & \sum_{k=2}^{m} \sum^{\prime} \frac{i^{l}}{(2 \pi)^{d}} \int_{|t| \leqslant T \sqrt{n}} e^{-i(t, x)} e^{-|t|^{2} / 2} e^{k|t|^{2} /(2 n)} \frac{n !}{(n-k) ! k_{3} ! \cdots k_{m+1} !} \\
& \times\left(\frac{\Delta_{3}\left(e_{t}\right)}{3 !}\left|\frac{t}{\sqrt{n}}\right|^{3}\right)^{k_{3}} \cdots\left(\frac{\Delta_{m+1}\left(e_{t}\right)}{(m+1) !}\left|\frac{t}{\sqrt{n}}\right|^{m+1}\right)^{k_{m+1}} d t
\end{aligned}
$$

где сумма $\sum^{\prime}$ берется по наборам $k_{3}, \ldots, k_{m+1}$, удовлетворяющим условиям (6). Здесь суммирование начинается с $k=2$ по причине, указанной выше. Справедлива цепочка неравенств:

$$
\begin{aligned}
& \left|R^{(m / 2)}\right| \leqslant \sum_{k=2}^{m} C_{n}^{k} \sum^{\prime} \frac{k !}{k_{3} ! \cdots k_{m+1} !} \frac{1}{(2 \pi)^{d}} \\
& \quad \times \int_{|t| \leqslant T \sqrt{n}} e^{-|t|^{2} / 2} e^{k|t|^{2} /(2 n)}\left(\frac{\delta_{3}}{3 !}\left|\frac{t}{\sqrt{n}}\right|^{3}\right)^{k_{3}} \cdots\left(\frac{\delta_{m+1}}{(m+1) !}\left|\frac{t}{\sqrt{n}}\right|^{m+1}\right)^{k_{m+1}} d t \\
& =\sum_{k=2}^{m} C_{n}^{k} \sum^{\prime} \frac{k !}{k_{3} ! \cdots k_{m+1} !} \frac{1}{(2 \pi)^{d}} \\
& \quad \times \int_{|t| \leqslant T \sqrt{n}} e^{-|t|^{2} / 2} e^{k|t|^{2} /(2 n)}\left|\frac{t}{\sqrt{n}}\right|^{m+2 k+1}\left(\frac{\delta_{3}}{3 !}\right)^{k_{3}} \cdots\left(\frac{\delta_{m+1}}{(m+1) !}\right)^{k_{m+1}}\left|\frac{t}{\sqrt{n}}\right|^{\eta} d t
\end{aligned}
$$

где $\eta=\eta\left(k_{1}, \ldots, k_{m+1}\right) \geqslant 0$. Так как $|t / \sqrt{n}| \leqslant T<1$, то правая часть последнего равенства не превосходит

$$
\begin{aligned}
& \sum_{k=2}^{m} C_{n}^{k} \sum^{\prime} \frac{k !}{k_{3} ! \cdots k_{m+1} !} \frac{1}{(2 \pi)^{d}} \\
& \quad \times \int_{|t| \leqslant T \sqrt{n}} e^{-|t|^{2} / 2} e^{k|t|^{2} /(2 n)}\left|\frac{t}{\sqrt{n}}\right|^{m+2 k+1}\left(\frac{\delta_{3}}{3 !}\right)^{k_{3}} \cdots\left(\frac{\delta_{m+1}}{(m+1) !}\right)^{k_{m+1}} d t \\
& \quad \leqslant \sum_{k=2}^{m} \frac{n^{k}}{k ! n^{(m+1) / 2+k}}\left(\sum_{s=3}^{m+1} \frac{\delta_{s}}{s !}\right)^{k} \frac{1}{(2 \pi)^{d}} \int_{E^{d}} e^{-(1-k / n)|t|^{2} / 2}|t|^{m+2 k+1} d t
\end{aligned}
$$

Сделаем замену переменных $t_{q}^{\prime}=t_{q} \sqrt{1-k / n}, q=1, \ldots, d$. Тогда выражение (7) не превосходит

$$
\begin{gathered}
\frac{1}{n^{(m+1) / 2}} \sum_{k=2}^{m} \frac{D_{3}^{k}}{k !}\left(\frac{n}{n-k}\right)^{(m+2 k+1+d) / 2} \frac{1}{(2 \pi)^{d}} \int_{E^{d}} e^{-|t|^{2} / 2}|t|^{m+2 k+1} d t \\
=\frac{1}{n^{(m+1) / 2}} \sum_{k=2}^{m} \frac{D_{3}^{k}}{k !}\left(\frac{n}{n-k}\right)^{(m+2 k+1+d) / 2} B_{m+2 k+1} .
\end{gathered}
$$


Далее, для каждого набора $k_{3}, \ldots, k_{m+1}, k_{3}+\cdots+k_{m+1}=k$, для которого выполняется условие (5), представим $e^{k|t|^{2} /(2 n)}$ в виде

$$
\sum_{j=0}^{J} \frac{(-1)^{j}}{j ! 2^{j}}\left(\frac{k}{n}\right)^{j}(i|t|)^{2 j}+r^{(J)},
$$

где $J=J\left(k_{3}, \ldots, k_{m+1}\right)$ - максимальное целое число, для которого $l / 2+J \leqslant m / 2+k$. Нетрудно проверить, что

$J=\left[\frac{m}{2}+k-\frac{l}{2}\right]=\left[\frac{m}{2}+k-\frac{3 k_{3}+\cdots+(m+1) k_{m+1}}{2}\right]=\left[\frac{m}{2}-\frac{k_{3}+\cdots+(m-1) k_{m+1}}{2}\right]$,

где [·] означает целую часть, и

$$
\left|r^{(J)}\right| \leqslant \frac{1}{(J+1) ! 2^{J+1}}\left(\frac{k}{n}\right)^{J+1}|t|^{2(J+1)} e^{k|t|^{2} /(2 n)} .
$$

Отметим также, что

$$
\frac{l}{2}+J=\frac{l}{2}+\left[\frac{m}{2}+k-\frac{l}{2}\right]= \begin{cases}\frac{m}{2}+k, & m-l \text { четно, } \\ \frac{m}{2}+k-\frac{1}{2}, & m-l \text { нечетно }\end{cases}
$$

откуда следует, что

$$
\frac{l}{2}+J+1 \geqslant \frac{m}{2}+k+\frac{1}{2}
$$

$$
0 \leqslant J \leqslant \frac{m-k}{2}
$$

Обозначим через $R^{(J)}$ сумму

$$
\begin{aligned}
R^{(J)}= & \sum_{k=1}^{m} \sum^{\prime \prime} \frac{i^{l}}{(2 \pi)^{d}} \int_{|t| \leqslant T \sqrt{n}} e^{-i(t, x)} e^{-|t|^{2} / 2} r^{(J)} \frac{n !}{(n-k) ! k_{3} ! \cdots k_{m+1} !} \\
& \times\left(\frac{\Delta_{3}\left(e_{t}\right)}{3 !}\left|\frac{t}{\sqrt{n}}\right|^{3}\right)^{k_{3}} \cdots\left(\frac{\Delta_{m+1}\left(e_{t}\right)}{(m+1) !}\left|\frac{t}{\sqrt{n}}\right|^{m+1}\right)^{k_{m+1}} d t
\end{aligned}
$$

где сумма $\sum^{\prime \prime}$ берется по наборам $k_{3}, \ldots, k_{m+1}$, для которых выполнено условие $(5)$, и каждое слагаемое убывает не медленнее, чем $1 / n^{(m+1) / 2}$.

Справедлива цепочка неравенств:

$$
\begin{aligned}
\left|R^{(J)}\right| \leqslant & \sum_{k=1}^{m} \sum^{\prime \prime} \frac{n !}{(n-k) ! k_{3} ! \cdots k_{m+1} !} \frac{1}{(2 \pi)^{d}} \\
& \times \int_{|t| \leqslant T \sqrt{n}}\left|r^{(J)}\right| e^{-|t|^{2} / 2}\left(\frac{\left|\Delta_{3}\left(e_{t}\right)\right|}{3 !}\left|\frac{t}{\sqrt{n}}\right|^{3}\right)^{k_{3}} \cdots\left(\frac{\left|\Delta_{m+1}\left(e_{t}\right)\right|}{(m+1) !}\left|\frac{t}{\sqrt{n}}\right|^{m+1}\right)^{k_{m+1}} d t \\
\leqslant & \sum_{k=1}^{m} C_{n}^{k} \sum^{\prime \prime} \frac{k !}{k_{3} ! \cdots k_{m+1} !} \frac{1}{(J+1) ! 2^{J+1}}\left(\frac{k}{n}\right)^{J+1}\left(\frac{\delta_{3}}{3 !}\right)^{k_{3}} \cdots\left(\frac{\delta_{m+1}}{(m+1) !}\right)^{k_{m+1}} \\
& \times \frac{1}{(2 \pi)^{d}} \int_{|t| \leqslant T \sqrt{n}} e^{k|t|^{2} /(2 n)} e^{-|t|^{2} / 2}|t|^{2(J+1)} \frac{|t|^{3 k_{3}+\cdots+(m+1) k_{m+1}}}{n^{\left(3 k_{3}+\cdots+(m+1) k_{m+1} / 2\right.}} d t \\
\leqslant & \sum_{k=1}^{m} C_{n}^{k} \sum^{\prime \prime} \frac{k !}{k_{3} ! \cdots k_{m+1} !}\left(\frac{\delta_{3}}{3 !}\right)^{k_{3}} \cdots\left(\frac{\delta_{m+1}}{(m+1) !}\right)^{k_{m+1}} \frac{k^{J+1}}{(J+1) ! 2^{J+1}} \frac{1}{n^{J+1+l / 2}} \\
& \times\left(\frac{n}{n-k}\right)^{J+1+\left(3 k_{3}+\cdots+(m+1) k_{m+1}\right) / 2+d / 2} \\
& \times \frac{1}{(2 \pi)^{d}} \int_{E^{d}} e^{-|t|^{2} / 2}|t|^{2(J+1)}|t|^{3 k_{3}+\cdots+(m+1) k_{m+1}} d t .
\end{aligned}
$$


Так как для моментов нормального распределения $B_{q} \leqslant B_{p}$ при $q \leqslant p$, то ввиду (10) последнее выражение не превосходит

$$
\begin{aligned}
\sum_{k=1}^{m} & C_{n}^{k} \sum^{\prime \prime} \frac{k !}{k_{3} ! \cdots k_{m+1} !}\left(\frac{\delta_{3}}{3 !}\right)^{k_{3}} \cdots\left(\frac{\delta_{m+1}}{(m+1) !}\right)^{k_{m+1}} \frac{k^{J+1}}{(J+1) ! 2^{J+1}} \frac{1}{n^{J+1+l / 2}} \\
& \times\left(\frac{n}{n-k}\right)^{(m-k) / 2+1+(m+2 k) / 2+d / 2} \frac{1}{(2 \pi)^{d}} \int_{E^{d}} e^{-|t|^{2} / 2}|t|^{m+2 k+m-k} d t \\
\leqslant & \sum_{k=1}^{m} \frac{n^{k} D_{3}^{k}}{k !} \frac{k^{J+1}}{(J+1) ! 2^{J+1}} \frac{1}{n^{J+1+l / 2}}\left(\frac{n}{n-k}\right)^{m+1+(k+d) / 2} \frac{1}{(2 \pi)^{d}} \\
& \times \int_{E^{d}} e^{-|t|^{2} / 2}|t|^{2 m+k} d t .
\end{aligned}
$$

В силу (9) правая часть последнего неравенства не превосходит

$$
\begin{gathered}
\sum_{k=1}^{m} \frac{D_{3}^{k}}{k !} \frac{k^{(m-k) / 2+1}}{2} \frac{n^{k}}{n^{(m+1) / 2+k}}\left(\frac{n}{n-k}\right)^{m+1+(k+d) / 2} \frac{1}{(2 \pi)^{d}} \int_{E^{d}} e^{-|t|^{2} / 2}|t|^{2 m+k} d t \\
=\frac{1}{n^{(m+1) / 2}} \sum_{k=1}^{m} \frac{D_{3}^{k}}{k !} \frac{k^{(m-k) / 2+1}}{2}\left(\frac{n}{n-k}\right)^{m+1+(k+d) / 2} B_{2 m+k} .
\end{gathered}
$$

Таким образом, справедливо представление

$$
\begin{aligned}
p_{n}(x)= & \varphi(x)+\sum_{k=1}^{m} \sum^{\prime \prime \prime} \frac{n !}{(n-k) ! k_{3} ! \cdots k_{m+1} !} \sum_{j=0}^{J} \frac{(-1)^{j}}{j ! 2^{j}}\left(\frac{k}{n}\right)^{j} \frac{i^{l+2 j}}{(2 \pi)^{d}} \\
& \times \int_{|t| \leqslant T \sqrt{n}} e^{-i(t, x)} e^{-|t|^{2} / 2}|t|^{2 j} \prod_{\ell=3}^{m+1}\left(\frac{i^{\ell} \Delta_{\ell}\left(e_{t}\right)}{\ell !}\left|\frac{t}{\sqrt{n}}\right|^{\ell}\right)^{k_{\ell}} d t \\
& +R+R^{(m / 2)}+R^{(J)},
\end{aligned}
$$

где сумма $\sum^{\prime \prime \prime}$ берется по наборам $k_{3}, \ldots, k_{m+1}, k_{3}+\cdots+k_{m+1}=k$, для которых выполнено условие (5), число $J$ определено в (8), оценка величины $R$ приведена в формулировке леммы, оценки величин $R^{(m / 2)}$ и $R^{(J)}$ даны выше.

В каждом из слагаемых в (11) заменим область интегрирования на $E^{d}$ и оценим возникающую при этом погрешность $R^{(T)}$ :

$$
\begin{aligned}
\left|R^{(T)}\right| \leqslant & \sum_{k=1}^{m} \sum^{\prime \prime \prime} \frac{n !}{(n-k) ! k_{3} ! \cdots k_{m+1} !} \sum_{j=0}^{J} \frac{1}{j ! 2^{j}}\left(\frac{k}{n}\right)^{j} \frac{1}{(2 \pi)^{d}} \\
& \times \int_{|t| \geqslant T \sqrt{n}} e^{-|t|^{2} / 2}|t|^{2 j r} \prod_{\ell=3}^{m+1}\left(\frac{i^{\ell} \Delta_{\ell}\left(e_{t}\right)}{\ell !}\left|\frac{t}{\sqrt{n}}\right|^{\ell}\right)^{k_{\ell}} d t \\
\leqslant & \sum_{k=1}^{m} \frac{n^{k}}{k !} \sum_{j=0}^{J} \frac{1}{j ! 2^{j}}\left(\frac{k}{n}\right)^{j} \frac{D_{k}}{(2 \pi)^{d}} \int_{|t| \geqslant T \sqrt{n}} e^{-|t|^{2} / 2}|t|^{2 j+2 m+k} d t \\
\leqslant & \sum_{k=1}^{m} \frac{n^{k}}{k !}\left(\frac{m-k}{2}+1\right) \frac{D_{k}}{(2 \pi)^{d}} \int_{|t| \geqslant T \sqrt{n}} e^{-|t|^{2} / 2}|t|^{3 m} d t .
\end{aligned}
$$

Отметим, что каждое из слагаемых в (12) при росте $n$ убывает экспоненциально быстро. Итак, плотность можно представить как

$$
p_{n}(x)=\varphi(x)+\sum_{k=1}^{m} \sum^{\prime \prime \prime} \frac{n !}{(n-k) ! k_{3} ! \cdots k_{m+1} !} \sum_{j=0}^{J} \frac{(-1)^{j}}{j ! 2^{j}}\left(\frac{k}{n}\right)^{j} \frac{i^{l+2 j}}{(2 \pi)^{d}}
$$




$$
\begin{aligned}
& \times \int_{E^{d}} e^{-i(t, x)} e^{-|t|^{2} / 2}|t|^{2 j} \prod_{\ell=3}^{m+1}\left(\frac{i^{\ell} \Delta_{\ell}\left(e_{t}\right)}{\ell !}\left|\frac{t}{\sqrt{n}}\right|^{\ell}\right)^{k_{\ell}} d t \\
& +R+R^{(m / 2)}+R^{(J)}+R^{(T)} .
\end{aligned}
$$

Сумма по $k=1, \ldots, m$ в правой части (13), дающая, вместе с функцией $\varphi(x)$, главную часть разложения, рассматривалась в [2]. Здесь мы получим в явном виде оценку остаточной части разложения.

Из результатов работы [2] и из сказанного выше следует справедливость следующего утверждения.

Теорема. В введенных обозначениях и предположениях, если абсолютный момент $\beta_{m+2}$ конечен, то при всех $n \geqslant \max (\nu, 2 m)$ у распределения $P_{n}$ нормированной суммь существует плотность $p_{n}(x)$ и для всех $x \in E^{d}$

$$
\begin{aligned}
& p_{n}(x)=\varphi(x)+\sum_{k=1}^{m} \sum_{k_{3}, \ldots, k_{m+1}} \frac{n !}{(n-k) ! k_{3} ! \cdots k_{m+1} !} \frac{1}{(3 !)^{k_{3}} \cdots((m+1) !)^{k_{m+1}}} \\
& \times \frac{\varphi(x)}{n^{\left(3 k_{3}+\cdots+(m+1) k_{m+1}\right) / 2}} \sum_{j=0}^{J} \frac{(-1)^{j}}{j ! 2^{j}}\left(\frac{k}{n}\right)^{j} \int_{E^{d}} \cdots \int_{E^{d}} H_{l}^{(2 j)}\left(u_{1}^{(3)}, u_{1}^{(3)}, u_{1}^{(3)}, \ldots, u_{k_{3}}^{(3)},\right. \\
& u_{k_{3}}^{(3)}, u_{k_{3}}^{(3)}, \ldots, \underbrace{u_{1}^{(m+1)}, \ldots, u_{1}^{(m+1)}}_{m+1}, \ldots, \underbrace{u_{k_{m+1}}^{(m+1)}, \ldots, u_{k_{m+1}}^{(m+1)}}_{m+1}) \\
& \times Q\left(d u_{1}^{(3)}\right) \cdots Q\left(d u_{k_{3}}^{(3)}\right) \cdots Q\left(d u_{1}^{(m+1)}\right) \cdots Q\left(d u_{k_{m+1}^{(m+1)}}^{(m+1)}+R,\right.
\end{aligned}
$$

где $Q=P-\Phi$, многомерные аналоги многочленов Чебышёва-Эрмита $H_{1}^{(2 j)}\left(h_{1}, \ldots, h_{1}\right)$ введены в работе [2] и сумма $\sum_{k_{3}, \ldots, k_{m+1}}$ берется по всем наборам $k_{3}, \ldots, k_{m+1}$ таким, ито $k_{3}+\cdots+k_{m+1}=k, 3 k_{3}+\cdots+(m+1) k_{m+1} \leqslant m+2 k, J=[m / 2-$ $\left.\left(k_{3}+\cdots+(m-1) k_{m+1}\right) / 2\right], u$

$$
\begin{aligned}
|R| \leqslant & \frac{\beta_{m+2}+\bar{\beta}_{m+2}}{(m+2) !} \sum_{k=1}^{m+1} \frac{D_{3, T}^{k-1}}{n^{(m+k-1) / 2}} \frac{B_{3(k-1)+m+2, n-k}}{k !}+\frac{D_{3, T}^{m+1}}{n^{(m+1) / 2}} \frac{B_{3(m+1), n-(m+1)}}{(m+1) !} \\
& +\frac{1}{n^{(m+1) / 2}} \sum_{k=2}^{m} \frac{D_{3}^{k}}{k !}\left(\frac{n}{n-k}\right)^{(m+2 k+1+d) / 2} B_{m+2 k+1} \\
& +\frac{1}{n^{(m+1) / 2}} \sum_{k=1}^{m} \frac{D_{3}^{k}}{k !} \frac{k^{(m-k) / 2+1}}{2}\left(\frac{n}{n-k}\right)^{m+1+(k+d) / 2} B_{2 m+k} \\
& +\frac{n^{d / 2}}{(2 \pi)^{d}} \alpha^{n-\nu}(T) \int_{|t| \geqslant T}|f(t)|^{\nu} d t+\frac{1}{(2 \pi)^{d}} \int_{|t| \geqslant T \sqrt{n}} e^{-|t|^{2} / 2} d t \\
& +\sum_{k=1}^{m} \frac{n^{k}}{k !}\left(\frac{m-k}{2}+1\right) \frac{D_{k}}{(2 \pi)^{d}} \int_{|t| \geqslant T \sqrt{n}} e^{-|t|^{2} / 2}|t|^{3 m} d t .
\end{aligned}
$$

Отметим, что слагаемые в остаточной части разложения, убывающие степенным образом, связаны только с моментами одномерных распределений, которые являются проекциями распределения $P$ на всевозможные многомерные векторы $e \in E^{d},|e|=1$.

\section{СПИСОК ЛИТЕРАТУРЫ}

1. Осмоловский И. Ю. О некоторых свойствах многомерных аналогов многочленов Чебышёва-Эрмита. - Теория вероятн. и ее примен., 2008, т. 53, в. 2, с. 373-378.

2. Сенатов В. В. О многомерных аналогах многочленов Чебышёва-Эрмита. - Теория вероятн. и ее примен., в печати. 
3. Сенатов B. В. Центральная предельная теорема: точность аппроксимации и асимптотические разложения. М.: Книжный Дом Либроком, 2009, 352 с.

Поступила в редакцию

17.IX.2008 